\title{
Simulación del Modelo de gestión para el control de riesgos y existencias del proceso de inventario en el almacén central de la Municipalidad Provincial de Huaura - Huacho 2017.
}

\section{Simulation of the Management model for the control of risks and stocks of the inventory process in the central warehouse of the Provincial Municipality of Huaura - Huacho 2017.}

Marycruz Lyly Huanca Trejo ${ }^{24}$, Giancarlos Joel López Marquez ${ }^{25}$, Julio Fabián Amado Sotelo ${ }^{26}$, Jaime Eduardo Gutiérrez Ascón ${ }^{27}$

\begin{abstract}
Resumen
Objetivo: Se determinó la manera en que el modelo de gestión para el control de riesgos asegura la disponibilidad del nivel de existencias del proceso de inventario en el almacén central. Materiales $\mathbf{y}$ métodos: El diseño de investigación fue no experimental de tipo transversal en su nivel causal. La población sujeto fue de 450 trabajadores y de objeto 64 artículos con una muestra sujeto de 142 al 95\% de confianza y la de objeto 8 artículos. En la investigación se usaron las técnicas de encuestas y análisis documental. Se aplicaron las herramientas de IPER, TOC, AMEF y simulación Monte Carlo con el software Crystal Ball. Resultados: La previsión del capital se encontró entre 124 877,52 a 151 535,92 soles al 95\% de confianza simulado 10000 veces. En donde, el costo fue sensible al papel bond A-4 quien aporta en un $86,9 \%$ en el capital. Además, con el modelo EOQ de productos múltiples se obtuvo un tamaño de lote alternativo de 559 millares en un tiempo óptimo de 15 días. Finalmente se logró de todos los tipos de familia una reducción del costo de 55 334,34 a 16 339,80 soles anuales de mantener y ordenar inventario siendo una disminución de 70,47\% que asegura la disponibilidad de existencias. Conclusiones: El modelo asegura la disponibilidad del nivel de existencias según Bustos y Chacón (2012), el cual concuerda con nuestro estudio al obtener $Q=155$ resmas para pedidos de papel oficio.

Palabras claves: Análisis de riesgos, teoría de restricciones, riesgo operacional, simulación, existencias.
\end{abstract}
Abstract
Objective: Determine the way in which the management model for risk control ensures the availability of the stock level of the inventory process in the central warehouse. Materials and methods: The research design was non-experimental of transversal type in its causal level. The subject population was of 450 workers and of object 64 articles with a sample subject of 142 to $95 \%$ confidence and that of object 8 articles. In the research, the techniques of surveys and documentary analysis were used. The tools of IPER, TOC, AMEF and Monte Carlo simulation were applied with the Crystal Ball software. Results: The capital forecast was between 124877.52 to 151535.92 soles at $95 \%$ confidence simulated 10000 times. Where, the cost was sensitive to bond paper A-4 who contributes $86.9 \%$ in capital. In addition, with the EOQ model of multiple products an alternative lot size of 559 packages was obtained in an optimal time of 15 days. Finally, a reduction in the cost of 55334.34 to 16339.80 soles per year was achieved for all family types, maintaining and ordering inventory, with a $70.47 \%$ decrease that ensures the availability of stocks. Conclusions: The model ensures availability of the stock level according to Bustos y Chacón (2012), which agrees with our study to obtain Q $=155$ reams for official paper orders.
Keywords: Risk analysis, restrictions theory, operational risk, simulation, stocks.

\footnotetext{
${ }^{24}$ Escuela Profesional de Ingeniería Industrial. Facultad de Ingeniería Industrial, Sistemas e Informática. Sánchez Carrión. Huacho - Perú.mhtrejo2@gmail.com.http://orcid.org/0000-0002-4663-4573. Telf. 953587419.

${ }^{25}$ Escuela de Ingeniería Industrial, Sistemas e Informática. Ing. Universidad Nacional José Faustino Sánchez Carrión. Huacho - Perú. https://orcid.org/0000-0002-7342-1549.

${ }^{26}$ Escuela de Ingeniería Industrial, Sistemas e Informática. Ing. Universidad Nacional José Faustino Sánchez Carrión. Huacho. Perú. http://orcid.org/0000-0001-9670-7796.

${ }^{27}$ Escuela de Ingeniería Industrial, Sistemas e Informática. Ing. Universidad Nacional José Faustino Sánchez Carrión. Huacho. Perú. http://orcid.org/0000-0003-4065-3359.
} 


\section{Introducción}

Un riesgo en la mayoría de casos puede ser provocado por múltiples causas y de producirse, suele tener uno o más efectos. Los riesgos según Hernández, Yelandy y Cuza (2013) poseen incertidumbre ya que pueden o no ocurrir, además ocasionan pérdida potencial, si el riesgo ocurre puede generar pérdidas no deseadas para la empresa.

Sin embargo, debido a ese problema, la gestión de riesgos es un tema abordado actualmente en muchas empresas e instituciones abarcándose en varios ámbitos como en procesos de inventarios, lo cual con el transcurrir del tiempo según Morón, Reyes y Urbina (2015) estas se han ido desarrollando y adaptando a los constantes cambios del entorno, teniendo en cuenta la conformación del origen de incertidumbres y riesgos dados del mal manejo de actividades, los cuales deben ser afrontados por los responsables de la administración, teniéndose en cuenta la percepción de los diferentes tipos de riesgo ya sean riesgos cambiarios (Lizarzaburu y Berggrun, 2013), riesgos financieros como los percibidos en diferentes periodos de compra on-line de pasajes aéreos (Fernandes da Silva, Fossati, y Affonso Silva, 2013), riesgos tecnológicos (Ulasio y Yépez, 2014), riesgos operacionales, entre otros.

De acuerdo a Ochoa y Rojas (2007) la gestión del riesgo se plantea como un proceso social complejo cuyo fin último es la reducción o la previsión y control permanente del riesgo de desastre en la sociedad, integrada y en consonancia al logro de pautas de desarrollo humano, económico, ambiental y territorial sostenibles. Además, Ortiz y Valencia (2017) considera actualmente que el rol que juega la competitividad de cualquier país está en el desarrollo de modelos de gestión específicos para estandarizar sus procesos y lograr niveles de eficacia y eficiencia en el cumplimiento de sus objetivos. Así mismo, Vanegas y Pardo (2014) argumentan que mediante un análisis de riesgos se permite planear, identificar, analizar, evaluar, controlar y monitorear los riesgos asociados con una actividad, función o proceso, para que la organización pueda reducir pérdidas y aumentar sus oportunidades enmarcadas en la mejora continua, lo cual COSO (2004) señala que siempre se evalúa en función desde una doble perspectiva probabilidad e impacto del que podrían causar dichos riesgos. Por ello Marín, Montes, Hernández y López (2010) mencionan que es necesario conocer cuál es el eslabón más débil que limita a un sistema a conseguir un mejor desempeño con relación a su meta. A su vez Gonzáles, Ortegón y Rivera (2003) menciona que todo se desarrolla en base a un mejoramiento continuo y visible en términos de utilidades, administrando el recurso más débil (la restricción) que exista para convertirlo en una ventaja. Sin embargo, López, Urrea y Navarro (2006) dan entender que cualquier organización está inmersa a cualquier restricción ya sea en temas como el mercadeo, la administración de la producción, las ventas, la estrategia, el manejo de personal, las finanzas y los proyectos resultando según Álvarez, Inche y Salvador (2004) que todo se acelere a un ritmo con un nivel adecuado sin sufrir consecuencias fatales.

En líneas generales y para toda clase de actividad económica se encuentra el denominado riesgo operacional, el cual se orienta de las decisiones que en el interior de la entidad se requieren diariamente, ya sea en relación a la producción, distribución, servicio al cliente, etc. (Llaguno, 2005; Mora y Gudiño, 2010; Soto, Stagg y Valente 2009). Por tanto, siempre su gestión deberá formar parte de la frecuente, así mismo operativa, gestión de mejora con la finalidad de conseguir siempre el ansiado progreso en eficiencia y en un buen ámbito empresarial. Para ello, según Nápoles, Silva y Marrero (2016) menciona que el Análisis Modal de Fallos y sus Efectos (AMFE) es una herramienta de gran utilidad que permite la valoración sistemática de las fallas potenciales en el diseño y la prestación de servicios ya sea de un producto, instrumento o proceso, evitando su aparición, controlando los efectos de las posibles fallas de variables significativas, de forma tal que nos permita prevenir productos o procesos defectuosos o inadecuados, antes de que estos lleguen al cliente. Además, González, Myer y Pachón (2017) detallan que todo ello permite un análisis integral del riesgo en cada etapa y, al mismo tiempo, plantear medidas adecuadas de tratamiento, orientadas a cada una de las variables, según el riesgo y la necesidad particular de cada sistema u organización. Así mismo, Izaguirre y Párraga (2017) detalla que el AMFE minimiza el costo de desarrollo y producción, reduce el consumo de tiempo para corregir fallas, captura el conocimiento de la organización, acentúa la prevención del problema, mejora la calidad y confiabilidad del producto y/o proceso. Este método, se determina identificando la ocurrencia, la severidad y la detectabilidad de los fallos, con el objetivo de resolver el número de prioridad de riesgo (NPR) (García et al., 2012). En consecuencia, Eppen, Gould, Schmidt, Moore, y Weatherford (2000) sostienen que también los modelos de simulación se utilizan a menudo para analizar una decisión bajo riesgo; esto es, un modelo en el cual el comportamiento de uno o más de los factores no se conoce con certeza. La simulación de Monte Carlo (Portuondo y Portuondo 2008), está presente en todos aquellos ámbitos en 
los que el comportamiento aleatorio o probabilístico desempeña un papel fundamental en estos casos como datos cinéticos o de materia prima (Serment, Mujica, Torres y Welti, 2012). Por otro lado, siempre existen riesgos en el proceso de inventario, ya que según Heizer y Render (2009) el inventario es uno de los activos más costosos de muchas empresas, el cual llega a simbolizar hasta un 50\% del capital total invertido es así que la inexistencia de un artículo puede paralizar la producción y dejar insatisfechos a los clientes. Así mismo, los modelos de inventarios (Izar, Ynzunza y Zermeño, 2015), han sido estudiados de manera intensiva durante los últimos tiempos, debido a que son importantes para un manejo adecuado de los bienes que se tienen en existencias. Otro detalle es que si no se tiene un inventario mínimo ( Álvarez y Cabrera, 2007) no garantiza la disponibilidad de inventario para atender al cliente. La función primordial está en la de proveer artículos de manera suficiente, de modo que si la demanda aumenta no haya faltantes, ya que representarían pérdidas en venta y muy posiblemente, una mala imagen ante los consumidores (Izar, Ynzunza, Castillo y Hernández, 2016).

Por lo tanto, el futuro de una organización puede estar ligado a algunos problemas que se derivan de un manejo inadecuado de sus inventarios. Dichos problemas podrían perjudicar la rentabilidad, el buen servicio y los costos, entre otros aspectos, por malas prácticas o prácticas conflictivas como el sobre ordenamiento o la disminución de existencias, quedando la organización poco preparada para responder a cambios abruptos externos, tales como alteraciones en la demanda y los precios (M. D. Arango, Adarme y Zapata, 2013; Valencia, Díaz y Correa, 2015; Valencia, Lambán y Royo, 2014).

Según Bustos y Chacón (2012) sostienen que precisamente, la compra y venta de artículos en la cantidad, calidad y tiempo de entrega deseado son un requisito que le permite a las empresas acumular los inventarios suficientes para satisfacer las demandas de sus clientes. En otras circunstancias Arango, Giraldo y Castrillón (2013) sostienen que lo que se busca siempre es un equilibrio que garantice la mínima inversión que permita el mejor nivel de servicio, teniendo la cantidad justa que se espera que el cliente requiera y con el objetivo primordial de optimizar el costo de mantener y ordenar inventario (Díaz y Pérez 2012).

Por ello, el objetivo de nuestro estudio es determinar la manera en que el modelo de gestión para el control de riesgos asegura la disponibilidad del nivel de existencias del proceso de inventario en el almacén central.

\section{Materiales y métodos}

El estudio de investigación fue de diseño no experimental puesto que está basado en hechos que ya ocurrieron. El tipo de investigación fue según su finalidad aplicada, según su alcance temporal transversal ya que se dio en un espacio de tiempo, según su profundidad explicativa y según su carácter de medida una investigación cuantitativa. En un nivel causal porque se pretende medir el comportamiento en función de causa - efecto al relacionar las variables, la gestión para el control de riesgos y existencias. La población sujeto fue de 450 trabajadores con muestra de 142 al $95 \%$ de confianza y la población objeto de 64 artículos con una muestra de 8 artículos a través del análisis ABC. En la investigación se usaron las siguientes técnicas: Encuestas y análisis documental y para el procesamiento de los datos las siguientes herramientas: la matriz IPER (Identificación de peligros y evaluación de riesgos) para la identificación de riesgos en el proceso de inventario y a través de una respuesta un plan de manejo del riesgo, TOC para identificar el eslabón más débil y mediante el modelo EOQ de productos múltiples elevar la restricción, AMEF para identificar el índice de prioridad del riesgo de los modos de fallo y dar tratamiento con acciones de mejoras y la simulación Monte Carlo con el software Crystal Ball.

\section{Resultados}

Para desarrollar una adecuada gestión para el control de riesgos se utilizaron las siguientes dimensiones los cuales fueron el análisis de riesgos, la teoría de restricciones, el riesgo operacional y la simulación. Análisis de riesgos. Con la ayuda de la técnica de recopilación de información del cuestionario se detectó los riesgos del proceso de inventario siguiendo una serie de pasos los cuales fueron: la identificación del proceso a través del software Bizagi Modeler 3.2 la identificación de riesgos en cada proceso desarrollándose en este paso que riesgos se presentan, dentro de ello las causas internas o externas del que provienen y las consecuencias que originan. Así mismo, la evaluación del riesgo con ayuda de las encuestas realizadas donde se identificó cuáles son las probabilidades y los impactos de los riesgos según las escalas de medidas cualitativas donde $b a j o=1$, medio $=2$ y alto $=3$ por lo que el trabajador evaluó según la materialización del riesgo y el efecto que causaría en caso de llegar a 
presentarse. También mediante el producto de las calificaciones dadas de la probabilidad y el impacto se determinó la prioridad del riesgo, permitiendo de este modo conocer en la matriz en qué zona del riesgo se encuentra.

Tabla 2. Prioridad del riesgo del proceso de inventario

\begin{tabular}{|c|c|c|c|c|c|c|c|}
\hline \multirow[b]{2}{*}{$\begin{array}{c}\text { Proces } \\
\quad 0\end{array}$} & \multirow[b]{2}{*}{ Riesgo } & \multicolumn{2}{|c|}{ Probabilidad } & \multicolumn{2}{|c|}{ Impacto } & \multirow{2}{*}{$\begin{array}{l}\text { Priorid } \\
\text { ad }\end{array}$} & \multirow{2}{*}{$\begin{array}{l}\text { Zona de } \\
\text { riesgo } \\
\text { inherente }\end{array}$} \\
\hline & & Calif. & Nivel & Calif. & Nivel & & \\
\hline \multirow{5}{*}{ 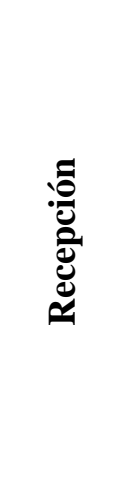 } & Faltantes de artículos & 3 & Alto & 3 & Alto & 9 & $\begin{array}{l}\text { Riesgo } \\
\text { inaceptable }\end{array}$ \\
\hline & Artículos dañados & 2 & $\begin{array}{c}\text { Medi } \\
\text { o }\end{array}$ & 2 & Medio & 4 & $\begin{array}{l}\text { Riesgo } \\
\text { moderado }\end{array}$ \\
\hline & Artículos caducados & 3 & Alto & 3 & Alto & 9 & $\begin{array}{l}\text { Riesgo } \\
\text { inaceptable }\end{array}$ \\
\hline & $\begin{array}{c}\text { Artículos con diferentes } \\
\text { características físicas a los } \\
\text { solicitado }\end{array}$ & 2 & $\begin{array}{l}\text { Medi } \\
\text { o }\end{array}$ & 2 & Medio & 4 & $\begin{array}{l}\text { Riesgo } \\
\text { moderado }\end{array}$ \\
\hline & $\begin{array}{l}\text { Retraso en las actividades que } \\
\text { realiza el trabajador }\end{array}$ & 3 & Alto & 1 & Bajo & 3 & $\begin{array}{l}\text { Riesgo } \\
\text { moderado }\end{array}$ \\
\hline \multirow{6}{*}{ 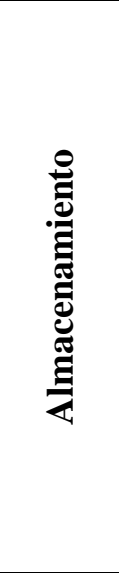 } & Pérdida de artículos & 2 & $\begin{array}{l}\text { Medi } \\
\text { o }\end{array}$ & 2 & Medio & 4 & $\begin{array}{l}\text { Riesgo } \\
\text { Moderado }\end{array}$ \\
\hline & Robo de artículos & 3 & Alto & 3 & Alto & 9 & $\begin{array}{l}\text { Riesgo } \\
\text { inaceptable }\end{array}$ \\
\hline & Espacio insuficiente & 3 & Alto & 3 & Alto & 9 & $\begin{array}{c}\text { Riesgo } \\
\text { inaceptable }\end{array}$ \\
\hline & $\begin{array}{c}\text { Pérdida de tiempo en } \\
\text { búsqueda }\end{array}$ & 3 & Alto & 2 & Medio & 6 & $\begin{array}{l}\text { Riesgo } \\
\text { importante }\end{array}$ \\
\hline & $\begin{array}{l}\text { Inconformidad en el registro } \\
\text { de entradas/salidas }\end{array}$ & 3 & Alto & 2 & Medio & 6 & $\begin{array}{l}\text { Riesgo } \\
\text { importante }\end{array}$ \\
\hline & $\begin{array}{c}\text { Costos de devaluación } \\
\text { (obsolescencia) de elementos } \\
\text { desclasificados }\end{array}$ & 2 & $\begin{array}{c}\text { Medi } \\
\text { o }\end{array}$ & 2 & Medio & 4 & $\begin{array}{l}\text { Riesgo } \\
\text { Moderado }\end{array}$ \\
\hline \multirow{5}{*}{ 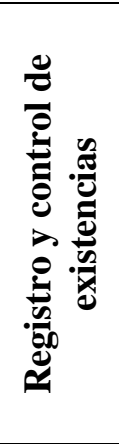 } & $\begin{array}{l}\text { Error en el registro de } \\
\text { artículos }\end{array}$ & 2 & $\begin{array}{c}\text { Medi } \\
\text { o }\end{array}$ & 2 & Medio & 4 & $\begin{array}{l}\text { Riesgo } \\
\text { Moderado }\end{array}$ \\
\hline & Cálculos incorrectos & 2 & $\begin{array}{c}\text { Medi } \\
\text { o }\end{array}$ & 2 & Medio & 4 & $\begin{array}{l}\text { Riesgo } \\
\text { Moderado }\end{array}$ \\
\hline & Re trabajo del personal & 2 & $\begin{array}{l}\text { Medi } \\
\text { o }\end{array}$ & 3 & Alto & 6 & $\begin{array}{l}\text { Riesgo } \\
\text { importante }\end{array}$ \\
\hline & $\begin{array}{l}\text { Demoras en realizar los } \\
\text { requerimientos }\end{array}$ & 3 & Alto & 3 & Alto & 9 & $\begin{array}{c}\text { Riesgo } \\
\text { inaceptable }\end{array}$ \\
\hline & Faltantes de artículos & 3 & Alto & 3 & Alto & 9 & $\begin{array}{c}\text { Riesgo } \\
\text { inaceptable }\end{array}$ \\
\hline & & & & & & & Continuaci \\
\hline \multirow{2}{*}{$\begin{array}{c}\text { Proces } \\
\text { o }\end{array}$} & & \multicolumn{2}{|c|}{ Probabilidad } & \multicolumn{2}{|c|}{ Impacto } & Priorid & Zona de \\
\hline & Riesgo & Calif. & Nivel & Calif. & Nivel & ad & $\begin{array}{l}\text { riesgo } \\
\text { inherente }\end{array}$ \\
\hline \multirow{4}{*}{ 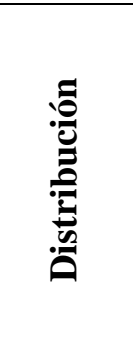 } & Insatisfacción del cliente & 2 & $\begin{array}{c}\text { Medi } \\
\text { o }\end{array}$ & 3 & Alto & 6 & $\begin{array}{l}\text { Riesgo } \\
\text { importante }\end{array}$ \\
\hline & $\begin{array}{l}\text { Demoras en la atención de } \\
\text { materiales a los clientes }\end{array}$ & 2 & $\begin{array}{c}\text { Medi } \\
\text { o }\end{array}$ & 2 & Medio & 4 & $\begin{array}{l}\text { Riesgo } \\
\text { Moderado }\end{array}$ \\
\hline & $\begin{array}{l}\text { Retraso en las actividades que } \\
\text { realizan las diferentes áreas }\end{array}$ & 2 & $\begin{array}{l}\text { Medi } \\
\text { o }\end{array}$ & 3 & Alto & 6 & $\begin{array}{l}\text { Riesgo } \\
\text { importante }\end{array}$ \\
\hline & $\begin{array}{c}\text { La nota de pedido está } \\
\text { incompleta }\end{array}$ & 2 & $\begin{array}{l}\text { Medi } \\
\text { o }\end{array}$ & 3 & Alto & 6 & $\begin{array}{l}\text { Riesgo } \\
\text { importante }\end{array}$ \\
\hline
\end{tabular}

Fuente: Elaboración propia con datos tomados de los trabajadores de la Municipalidad Provincial de 


\begin{tabular}{ccccccc} 
Error al elegir los artículos & 3 & Alto & 3 & Alto & 9 & $\begin{array}{c}\text { Riesgo } \\
\text { inaceptable }\end{array}$ \\
$\begin{array}{c}\text { Acondicionar el pedido con } \\
\text { mayor o menor cantidad }\end{array}$ & 1 & Bajo & 2 & Medio & 2 & $\begin{array}{c}\text { Riesgo } \\
\text { tolerable }\end{array}$ \\
$\begin{array}{c}\text { Deterioro de los artículos al } \\
\text { momento de ser trasladados }\end{array}$ & 2 & $\begin{array}{c}\text { Medi } \\
\text { o }\end{array}$ & 2 & Medio & 4 & $\begin{array}{c}\text { Riesgo } \\
\text { Moderado }\end{array}$ \\
\hline
\end{tabular}

Por otro lado, según la sumatoria total de todos los niveles de riesgos inherentes presentados anteriormente se tuvo que el $43,5 \%$ son riesgos tolerables, $26,09 \%$ son riesgos importantes, $39,13 \%$ son riesgos moderados y el 30,43\% son riesgos inaceptables. Por lo tanto se seleccionó por prioridad a los riesgos inaceptables e importantes, ya que requieren una acción inmediata. Por consiguiente, se dio una respuesta realizando un plan de manejo del riesgo en la cual se da opciones de aceptar, evitar, reducir y compartir el riesgo existente, un control propuesto o acciones a tomar, que tipo de control tomar ya sea preventivo o correctivo y el responsable que estará a cargo de llevar a cabo dicho control. Dentro del control propuesto se propuso establecer un tiempo de reposición de materiales y realizar los requerimientos necesarios con el modelo EOQ para productos múltiples, con esto permitir un apropiado abastecimiento de materiales y así reducir el riesgo llevando un mejor control dentro del proceso de inventario.

Teoría de restricciones. Analizando el proceso de distribución que se realiza cada día en el área de almacén y teniendo en cuenta la clasificación por familia grupo A: suministros de impresora, artículos de oficina y artículos de limpieza se tuvo en cuenta los tiempos en cada proceso, evaluándose si existe un stock cero en el cual se presentó la restricción, siendo así el tiempo de reposición de artículos 7 días más el tiempo del proceso de control de existencias equivalente a 2 minutos. Además la demanda mensual desde setiembre del 2016 - agosto del 2017. Siendo así, se siguió los siguientes pasos:

Paso 1. Identificar la restricción del sistema. Por no tener un apropiado sistema de control de reposición de los artículos. Se identificó como restricción al proceso de verificación y control de existencias con una capacidad utilizada de 391986 minutos (la suma total de multiplicar el tiempo empleado por cada producto en cada proceso por la demanda anual de cada recurso) para satisfacer la totalidad de la demanda. Como este recurso excede los 126720 minutos anuales (8h/día x $60 \mathrm{~min} / \mathrm{h} \mathrm{x}$ 22 días x 12 meses) disponibles de capacidad y los demás requieren menos tiempo, entonces este es la limitación.

Tabla 3. Capacidad utilizada de cada artículo por proceso

\begin{tabular}{|c|c|c|c|c|c|c|c|c|c|c|c|}
\hline \multirow{3}{*}{ Productos } & \multirow{3}{*}{$\begin{array}{c}\text { D } \\
\text { (anua } \\
\text { l) }\end{array}$} & \multicolumn{10}{|c|}{ Procesos } \\
\hline & & $\begin{array}{r}\text { 1.Fo } \\
\text { ó } \\
\text { pe }\end{array}$ & $\begin{array}{l}\text { mulaci } \\
\text { del } \\
\text { dido }\end{array}$ & $\begin{array}{r}2 . \text { Ver } \\
\text { n y c } \\
\text { exist }\end{array}$ & $\begin{array}{l}\text { ificació } \\
\text { ontrol } \\
\text { de } \\
\text { encias }\end{array}$ & $\begin{array}{r}\text { 3.Au } \\
\text { ó } \\
\text { des }\end{array}$ & $\begin{array}{l}\text { orizaci } \\
\text { de } \\
\text { acho }\end{array}$ & $\begin{array}{l}\text { 4.Acc } \\
\text { ami } \\
\text { mat }\end{array}$ & $\begin{array}{l}\text { Idicion } \\
\text { to de } \\
\text { riales }\end{array}$ & $\begin{array}{r}\text { 5.Ent } \\
\text { mat }\end{array}$ & $\begin{array}{l}\text { ega de } \\
\text { riales }\end{array}$ \\
\hline & & $\begin{array}{c}\text { Tie } \\
\text { mp } \\
\text { o } \\
(\mathrm{mi} \\
\mathrm{n})\end{array}$ & Cap. & $\begin{array}{c}\text { Tie } \\
\text { mpo } \\
(\mathrm{min} \\
\text { ) }\end{array}$ & Cap. & $\begin{array}{c}\text { Tie } \\
\text { mpo } \\
(\mathrm{mi} \\
\mathrm{n})\end{array}$ & Cap. & $\begin{array}{c}\text { Tie } \\
\text { mpo } \\
(\mathrm{min} \\
\text { ) }\end{array}$ & Cap. & $\begin{array}{c}\text { Tie } \\
\text { mpo } \\
(\mathrm{mi} \\
\mathrm{n})\end{array}$ & Cap. \\
\hline $\begin{array}{l}\text { Tinta Epson } \\
\text { negro }\end{array}$ & 126 & 2 & 252 & 2 & 252 & 1 & 126 & 5 & 630 & 3 & 378 \\
\hline $\begin{array}{l}\text { Cinta Epson } \\
\text { Fx-890 }\end{array}$ & 214 & 2 & 428 & 2 & 428 & 1 & 214 & 5 & 1070 & 3 & 642 \\
\hline $\begin{array}{l}\text { Tinta Epson } \\
\text { L210 cyan }\end{array}$ & 52 & 2 & 104 & 422 & 21944 & 1 & 52 & 5 & 260 & 3 & 156 \\
\hline $\begin{array}{l}\text { Tinta Epson } \\
\text { L210 magenta }\end{array}$ & 52 & 2 & 104 & 422 & 21944 & 1 & 52 & 5 & 260 & 3 & 156 \\
\hline $\begin{array}{l}\text { Tinta Epson } \\
\text { L350 amarillo }\end{array}$ & 51 & 2 & 102 & 2 & 102 & 1 & 51 & 5 & 255 & 3 & 153 \\
\hline
\end{tabular}




\begin{tabular}{lrrrrrrrrrrr}
\hline $\begin{array}{l}\text { Papel bond A- } \\
4\end{array}$ & 4014 & 2 & 8028 & 2 & 8028 & 1 & 4014 & 5 & 20070 & 3 & 12042 \\
Archivador & 800 & 2 & 1600 & 422 & 337 & 1 & 800 & 5 & 4000 & 3 & 2400 \\
$\begin{array}{l}\text { Carro de } \\
\text { limpieza }\end{array}$ & 4 & 2 & 8 & 422 & 1688 & 1 & 4 & 5 & 20 & 3 & 12 \\
$\begin{array}{l}\text { Capacidad } \\
\text { utilizada }\end{array}$ & & & 10626 & & $\mathbf{3 9 1}$ & & 5313 & 26565 & 15939 \\
$\begin{array}{l}\text { Capacidad } \\
\text { disponible }\end{array}$ & & & 126 & & $\mathbf{9 8 6}$ & & 126 & 126 & & 126 & \\
\hline
\end{tabular}

Fuente: Elaboración propia con datos tomados del almacén de la Municipalidad Provincial de Huaura

Paso 2. Explotar la restricción. La manera de explotar la restricción se realizó analizando las capacidades de cada artículo y dándole la mayor prioridad a la familia del artículo que tiene la mayor capacidad. En nuestro caso el artículo de archivador es el que tiene la mayor prioridad con una capacidad disponible de 337600 minutos, por lo tanto se analizó en primer lugar la familia de artículos de oficina. Por otro lado, las series de consumo analizadas por familia de artículos mostraron el comportamiento anual de estos, para lo cual con el modelo EOQ de productos múltiples ordenados y entregados de manera conjunta se determinó la cantidad óptima a pedir y el tiempo de reposición entre pedidos, teniendo en cuenta los costos asociados al manejo de inventario que posee el almacén como: el costo de ordenar (S) de 12, 02 soles/orden el cual se determinó del costo total anual de gestión para realizar la actividad con un total de 1166 soles entre 97 de órdenes de compra realizadas en el periodo y el de almacenar inventario $(\mathrm{H})$ se determinó teniendo en cuenta el costo de oportunidad en el cual incurre la institución al mantener artículos en almacén por la tasa en la cual se estimó a un 6,30\% anual, según la tasa anual para depósitos a plazo fijo de la caja Sullana con la cual la institución mantiene relaciones comerciales.

Teniendo en cuenta todo ello se analizó el costo total anual del inventario correspondiente a la política actual y el costo total anual para el tamaño de lote alternativo, como también el tiempo entre pedidos para cada tipo de familia de artículos, los cuales son los siguientes:

\section{Familia de artículos de oficina:}

- Demanda: $\mathrm{D}_{\mathrm{A}}=800$ unid/año, $\mathrm{D}_{\mathrm{P}}=4014$ millares/año

- Tamaño de lote actual: $\mathrm{Q}_{A}=400$ unid/año, $\mathrm{Q}_{\mathrm{P}}=2600$ millares/año

- Costo especifico de ordenar cada producto: $\mathrm{S}_{\mathrm{A}}=12,02$ soles, $\mathrm{S}_{\mathrm{P}}=12,02$ soles

- Costo de mantener inventario: $\mathrm{h}=6,30 \%$

- Costo unitario: $\mathrm{C}_{\mathrm{A}}=4,07$ soles, $\mathrm{C}_{\mathrm{P}}=12,40$ soles

El costo total anual de acuerdo a la política actual aplicando la fórmula es lo siguiente:

(1)

$\mathrm{C}=\frac{\mathrm{Q}}{2}(\mathrm{H})+\frac{\mathrm{D}}{\mathrm{Q}}(\mathrm{S})$

$\mathrm{C}_{\mathrm{G}}=\mathrm{S} / .76,04+\mathrm{S} / .1032,56=\mathrm{S} / .1108,60$

El costo total anual de la familia de artículos de oficina para el tamaño de lote alternativo.

Debido a que los dos artículos se incluyen en cada pedido, el costo combinado de ordenar es lo siguiente:

(2)

$S^{*}=S+S_{A}+S_{P}$

$\mathrm{S}^{*}=12,02+12,02=24,04$ soles por pedido

La frecuencia optima del pedido:

$\mathrm{n}^{*}=\sqrt{\frac{\mathrm{D}_{\mathrm{A}} \mathrm{hC}_{\mathrm{A}}+\mathrm{D}_{\mathrm{P}} \mathrm{hC}_{\mathrm{P}}}{2 \mathrm{~S}^{*}}}$ 
$\mathrm{n}^{*}=\sqrt{\frac{800 \times(0,063 \times 4,07)+4014 \times(0,063 \times 12,40)}{2 \times 24,04}}=8,34 \cong 8$ pedidos/año

Costo anual de ordenar:

$C_{S}=S^{*} n$

(4)

$\mathrm{C}_{\mathrm{S}}=24,04 \times 8=192,32$ soles

Costo anual de ordenar y mantener inventario:

$\mathrm{C}_{\mathrm{T}}=\frac{\mathrm{D}_{\mathrm{A}} \mathrm{hC}_{\mathrm{A}}}{2 \mathrm{n}}+\frac{\mathrm{DPhC}_{\mathrm{P}}}{2 \mathrm{n}}+\mathrm{S}^{*} \mathrm{n}$

(5)

$\mathrm{C}_{\mathrm{T}}=\frac{800 \times(0,063 \times 4,07)}{2 \times 8}+\frac{4014 \times(0,063 \times 12,40)}{2 \times 8}+192,32=\mathrm{S} / .401,12$

Tabla 4. Tamaños de lote y costos de pedidos de la familia de artículos de oficina

\begin{tabular}{lrr} 
& Archivador & Papel Bond A-4 \\
\hline Demanda por año (D) & 800 & 4014 \\
Frecuencia del pedido $\left(\mathrm{n}^{*}\right)$ & $8 / a$ ño & $8 /$ año \\
Tamaño óptimo del pedido $\mathrm{Q}^{*}=\left(\mathrm{D} / \mathrm{n}^{*}\right)$ & 100 & 502 \\
Inventario de ciclo $=\left(\mathrm{Q}^{*} / 2\right)$ & 50 & 251 \\
Costo anual de mantener inventario & $\mathrm{S} / .12,82$ & $\mathrm{~S} / .195,98$ \\
Tiempo de flujo promedio $=\left(\mathrm{Q}^{*} / 2 \mathrm{D}\right)$ & 17 días & 17 días \\
\hline
\end{tabular}

Fuente: Elaboración propia con datos a través de las ecuaciones (3) y (5)

Interpretación: El modelo EOQ de productos múltiples ordenados y entregados de manera conjunta, nos determinó un tamaño de lote de pedido óptimo $\left(\mathrm{Q}^{*}\right)$ para: $Q^{*}{ }_{A}=100$ unidades y $Q^{*}{ }_{P}=502$ millares con un tiempo entre pedidos de 0.0625 anual, además teniendo en cuenta que se trabaja 22 días al mes un total de 17 días y un total de costo anual óptimo de S/.401,12.

De este modo, la decisión que se incurre es que siendo el tamaño de lote que realiza actualmente el área de almacén 400 unidades de archivador y 2600 millares de papel bond A-4 cada 6 meses a un costo total de 1 108,60 soles anuales de mantener y ordenar inventario es una opción más cara que la opción del tamaño de lote alternativo con un costo total de 401,12 soles anuales. De esta manera se lograría una reducción del $63,82 \%$ del costo de inventario de 1108.60 a 401,12 soles anuales con un tiempo de reposición de pedidos manejado cada 17 días para optimizar el flujo de dinero de la institución.

Familia de suministros de impresora:

- Demanda: $\mathrm{D}_{\mathrm{TCYAN}}=52$ unid/año, $\mathrm{D}_{\mathrm{TMAG}}=52$ unid/año, $\mathrm{D}_{\mathrm{CFX}-890}=214$ unid/año, $\mathrm{D}_{\mathrm{TNEG}}=126$ unid/año, $\mathrm{D}_{\text {TAM }}=51$ unid/año

- Tamaño de lote actual: $\mathrm{Q}_{\mathrm{TCYAN}}=26$ unid/año, $\mathrm{Q}_{\mathrm{TMAG}}=26$ unid/año, $\mathrm{Q}_{\mathrm{CFX}-890}=150$ unid/año, $\mathrm{Q}_{\mathrm{TNEG}}=$ 78 unid/año, $\mathrm{Q}_{\text {TAM }}=26$ unid/año

- Costo especifico de ordenar cada producto: $\mathrm{S}_{\mathrm{TCYAN}}=12,02$ soles, $\mathrm{S}_{\mathrm{TMAG}}=12,02$ soles, $\mathrm{S}_{\mathrm{CFX}-890}=12,02$ soles, $\mathrm{S}_{\mathrm{TNEG}}=12,02$ soles, $\mathrm{S}_{\mathrm{TAM}}=12,02$ soles

- Costo de mantener inventario: $\mathrm{h}=6,30 \%$

- Costo unitario: $\mathrm{C}_{\mathrm{TCYAN}}=75$ soles, $\mathrm{C}_{\mathrm{TMAG}}=75$ soles, $\mathrm{C}_{\mathrm{CFX}-890}=30$ soles, $\mathrm{C}_{\mathrm{TNEG}}=75$ soles, $\mathrm{C}_{\mathrm{TAM}}=75$ soles

El costo total anual de acuerdo a la política actual:

$\mathrm{C}_{\mathrm{G}}=\mathrm{S} / .85,53+\mathrm{S} / .85,53+\mathrm{S} / .158,90+\mathrm{S} / .203,89+\mathrm{S} / .85,07=\mathrm{S} / .618,92$

El costo total anual de la familia de suministros de impresora para el tamaño de lote alternativo.

Debido a que los 5 artículos se incluyen en cada pedido, el costo anual de ordenar y mantener inventario: $\mathrm{C}_{\mathrm{T}}=\frac{52 \mathrm{x}(4,73)}{2 \times 4}+\frac{52 \times(4,73)}{2 \times 4}+\frac{214 \mathrm{x}(1,89)}{2 \times 4}+\frac{126 \times(4,73)}{2 \times 4}+\frac{51 \times(4,73)}{2 \times 4}+240,40=\mathrm{S} / .457,10$ 
Tabla 5. Tamaños de lote y costos de pedidos de la familia de suministros de impresora

\begin{tabular}{|c|c|c|c|c|c|}
\hline & $\begin{array}{c}\text { Tinta } \\
\text { Epson } \\
\text { L210 cyan }\end{array}$ & $\begin{array}{c}\text { Tinta } \\
\text { Epson L210 } \\
\text { magenta }\end{array}$ & $\begin{array}{c}\text { Cinta } \\
\text { Epson Fx- } \\
890\end{array}$ & $\begin{array}{l}\text { Tinta } \\
\text { Epson } \\
\text { negro }\end{array}$ & $\begin{array}{c}\text { Tinta } \\
\text { Epson } \\
\text { L350 } \\
\text { amarillo } \\
\end{array}$ \\
\hline Demanda por año (D) & 52 & 52 & 214 & 126 & 51 \\
\hline Frecuencia del pedido(n*) & 4/año & 4/año & 4/año & 4/año & 4/año \\
\hline $\begin{array}{l}\text { Tamaño óptimo del pedido } Q^{*}= \\
\left(D / n^{*}\right)\end{array}$ & 13 & 13 & 54 & 32 & 13 \\
\hline Inventario de ciclo $=\left(Q^{*} / 2\right)$ & 7 & 7 & 27 & 16 & 7 \\
\hline $\begin{array}{l}\text { Costo anual de mantener } \\
\text { inventario }\end{array}$ & S/.30,75 & S/.30,75 & $\mathrm{S} / .50,56$ & $\mathrm{~S} / .74,50$ & $\mathrm{~S} / .30,15$ \\
\hline $\begin{array}{l}\text { Tiempo de flujo promedio= } \\
\left(Q^{*} / 2 D\right)\end{array}$ & 33 días & 33 días & 33 días & 33 días & 33 días \\
\hline
\end{tabular}

Fuente: Elaboración propia con datos a través de la ecuaciones (3) y (5)

Interpretación: El modelo EOQ de productos múltiples ordenados y entregados de manera conjunta nos determinó un tamaño de lote de pedido óptimo $\left(\mathrm{Q}^{*}\right)$ para: $Q^{*}{ }_{\text {TCYAN }}=13$ unidades, $Q^{*}{ }_{\text {TMAG }}=13$ unidades, $Q_{C F X-890}^{*}=54$ unidades, $Q^{*}{ }_{T N E G}=32$ unidades y $Q^{*}{ }_{T A M}=13$ unidades con un tiempo entre pedidos de 0.125 anual, además teniendo en cuenta que se trabaja 22 días al mes un total de 33 días y un total de costo anual óptimo de S/.457,10.

De este modo, la decisión que se incurre es que siendo el tamaño de lote que realiza actualmente el área de almacén 26 unidades de tinta Epson L210 cyan, 26 unidades de tinta Epson L210 magenta, 150 unidades de cinta Epson Fx-890, 78 unidades de tinta Epson negro y 26 unidades de tinta Epson L350 amarillo cada 6 meses a un costo total de 618,92 soles anuales de mantener y ordenar inventario es una opción más cara que la opción del tamaño de lote alternativo con un costo total de 457,10 soles anuales. De esta manera se lograría una reducción del 26,15\% del costo de inventario de 618,92 a 457,10 soles anuales con un tiempo de reposición de pedidos manejado cada 33 días para optimizar el flujo de dinero de la institución.

\section{Familia de artículos de limpieza}

\section{Carro de limpieza}

- Demanda anual: 4 unidades

- Costo unitario: 1800 soles

- Tamaño de lote actual: 4 unidades

- Costo de almacenamiento(H): 113,40 soles

- Costo de ordenar(S): 12,02 soles

El costo total anual de carro de limpieza de acuerdo a la política actual:

$\mathrm{C}=\frac{4 \text { unid }}{2}(113,40)+\frac{4 \text { unid }}{4 \text { unid }}(12,02)=\mathrm{S} / .238,82$

El costo total anual de la familia de artículos de limpieza para el tamaño de lote alternativo.

El costo anual de ordenar y mantener inventario:

$\mathrm{C}_{\mathrm{T}}=\frac{4 \times(113,40)}{2 \times 4}+48,08=\mathrm{S} / .104,78$

Tabla 6: Tamaños de lote y costos de pedidos de la familia de artículos de limpieza

Demanda por año (D)

Frecuencia del pedido(n*)

Tamaño óptimo del pedido $\mathrm{Q}^{*}=\left(\mathrm{D} / \mathrm{n}^{*}\right)$

Inventario de ciclo $=\left(\mathrm{Q}^{*} / 2\right)$

Costo anual de mantener inventario

Tiempo de flujo promedio $=\left(\mathrm{Q}^{*} / 2 \mathrm{D}\right)$
Carro de limpieza

4/pedido

1

S $/ .56,70$

33 días

Fuente: Elaboración propia con datos a través de las ecuaciones (3) y (5) 


\section{Interpretación:}

El modelo EOQ de productos múltiples ordenados y entregados de manera conjunta nos determinó un tamaño de lote de pedido óptimo $\left(\mathrm{Q}^{*}\right): Q^{*}{ }_{C L}=1$ unidad con un tiempo entre pedidos de 0,125 anual, además teniendo en cuenta que se trabaja 22 días al mes un total de 33 días y un total de costo anual óptimo de S/.104,78. De este modo, la decisión que se incurre es que siendo el tamaño de lote que realiza actualmente el área de almacén 4 unidades de carro de limpieza a un costo total de 238,82 soles anuales de mantener y ordenar inventario es una opción más cara que la opción del tamaño de lote alternativo con un costo total de 104,78 soles anuales. De esta manera se lograría una reducción del $56,13 \%$ del costo de inventario de 238,82 a 104,78 soles anuales con un tiempo de reposición de pedidos manejado cada 33 días para optimizar el flujo de dinero de la institución.

Por ende para analizar el análisis del throughput se tuvo en cuenta que el almacén de la institución solamente brinda servicios de almacenamiento y distribución de artículos para las distintas áreas del municipio, por lo tanto en el cálculo del throughput los precios de las ventas se consideran cero quedando los costos variables igual al throughput negativo.

Throughput $=P V-C V$

(6)

Throughput $=0-\mathrm{CV}$

- Throughput $=$ Costos variables

Throughput negativo de acuerdo a la política actual de todas las familias de artículos:

- Throughput $=Q C_{u}+C_{\text {ordenar }}+C_{\text {Mantener }}$

- Throughput $_{\text {Total }}=(400 \times 4,07+2600 \times 12,40+1108,60)+(26 \times 75+26 \times 75+150 \times 30+78 \times$

$75+26 \times 75+618,92)+(4 \times 1800+238,82)=\mathrm{S} / .55334,34$

Throughput negativo alternativo de todas las familias de artículos:

- Throughput $_{\text {Total }}=(100 \times 4,07+502 \times 12,40+401,12)+(13 \times 75+13 \times 75+54 \times 30+32 \times 75$ $+13 \times 75+457,10)+(1 \times 1800+104,78)=S / .16339,80$

Por consiguiente, disminuyó el throughput negativo de 55 334,34 soles a 16 339,80 soles al optimizar el tiempo y el tamaño de lote de pedido, lo cual esto representa una disminución de 70,47\%.

Paso 3. Subordinar la restricción. Se establecieron los tiempos correctos de los procesos del almacén, ya que al aplicar el modelo de reposición de artículos con el propósito de mejorar la disponibilidad de las existencias, el tiempo de reposición entre pedidos según el consumo anual se tuvo de la tabla 9 , en el registro y control de existencias una capacidad de 10626 minutos por debajo de la capacidad disponible de 126720 minutos, resultándose de esta manera que los otros procesos tengan una variación mínima de utilización con respecto a esta.

Paso 4. Elevar la restricción. Es necesario aplicar el modelo EOQ para artículos múltiples que permita un adecuado control de existencias, así de esta manera estandarizar el proceso y obtener mejoras del mismo, lográndose un adecuado abastecimiento y satisfacción de las áreas usuarias de la institución. Así mismo, mejorar la disponibilidad de dinero de la institución.

Paso 5. Se logró romper la restricción. Al evaluar nuevamente la capacidad utilizada, pero el proceso de acondicionamiento de materiales tiene una utilización mayor que los demás procesos, por ende se recomienda que se realice una distribución de personal para la mejora de esta; consecuentemente el ciclo de mejora continua.

T 
abla 7: Subordinación de los procesos a la restricción

\begin{tabular}{|c|c|c|c|c|c|c|c|c|c|c|c|}
\hline \multirow{3}{*}{ Productos } & \multirow{3}{*}{$\begin{array}{c}\text { D } \\
\text { (anua } \\
\text { l) }\end{array}$} & \multicolumn{10}{|c|}{ Procesos } \\
\hline & & $\begin{array}{r}\text { 1.Fol } \\
\text { ór } \\
\text { pe }\end{array}$ & $\begin{array}{l}\text { mulaci } \\
\text { del } \\
\text { dido }\end{array}$ & $\begin{array}{c}2 . \text { Ver } \\
\text { n y } \\
\text { exis }\end{array}$ & $\begin{array}{l}\text { fficació } \\
\text { ontrol } \\
\text { le } \\
\text { encias }\end{array}$ & $\begin{array}{r}\text { 3.Au } \\
\text { o } \\
\text { des }\end{array}$ & $\begin{array}{l}\text { rizaci } \\
\text { de } \\
\text { acho }\end{array}$ & $\begin{array}{l}\text { 4.Aco } \\
\text { ami } \\
\text { mat }\end{array}$ & $\begin{array}{l}\text { ndicion } \\
\text { nto de } \\
\text { riales }\end{array}$ & $\begin{array}{r}\text { 5.En } \\
\text { mat }\end{array}$ & $\begin{array}{l}\text { ega de } \\
\text { riales }\end{array}$ \\
\hline & & $\begin{array}{c}\text { Tie } \\
\text { mp } \\
\text { o } \\
(\mathrm{mi} \\
\mathrm{n})\end{array}$ & Cap. & $\begin{array}{c}\text { Tie } \\
\text { mpo } \\
(\mathrm{min} \\
\text { ) }\end{array}$ & Cap. & $\begin{array}{c}\text { Tie } \\
\text { mpo } \\
(\mathrm{mi} \\
\mathrm{n})\end{array}$ & Cap. & $\begin{array}{c}\text { Tie } \\
\text { mpo } \\
(\mathrm{min} \\
\text { ) }\end{array}$ & Cap. & $\begin{array}{c}\text { Tie } \\
\text { mpo } \\
(\mathrm{mi} \\
\mathrm{n})\end{array}$ & Cap. \\
\hline $\begin{array}{l}\text { Tinta Epson } \\
\text { negro }\end{array}$ & 126 & 2 & 252 & 2 & 252 & 1 & 126 & 5 & 630 & 3 & 378 \\
\hline $\begin{array}{l}\text { Cinta Epson } \\
\text { Fx-890 }\end{array}$ & 214 & 2 & 428 & 2 & 428 & 1 & 214 & 5 & 1070 & 3 & 642 \\
\hline $\begin{array}{l}\text { Tinta Epson } \\
\text { L210 cyan }\end{array}$ & 52 & 2 & 104 & 2 & 104 & 1 & 52 & 5 & 260 & 3 & 156 \\
\hline $\begin{array}{l}\text { Tinta Epson } \\
\text { L210 magenta }\end{array}$ & 52 & 2 & 104 & 2 & 104 & 1 & 52 & 5 & 260 & 3 & 156 \\
\hline $\begin{array}{l}\text { Tinta Epson } \\
\text { L350 amarillo }\end{array}$ & 51 & 2 & 102 & 2 & 102 & 1 & 51 & 5 & 255 & 3 & 153 \\
\hline $\begin{array}{l}\text { Papel bond A- } \\
4\end{array}$ & 4014 & 2 & 8028 & 2 & 8028 & 1 & 4014 & 5 & 20070 & 3 & 12042 \\
\hline Archivador & 800 & 2 & 1600 & 2 & 1600 & 1 & 800 & 5 & 4000 & 3 & 2400 \\
\hline $\begin{array}{l}\text { Carro de } \\
\text { limpieza }\end{array}$ & 4 & 2 & 8 & 2 & 8 & 1 & 4 & 5 & 20 & 3 & 12 \\
\hline $\begin{array}{l}\text { Capacidad } \\
\text { utilizada }\end{array}$ & & & 10626 & & 10626 & & 5313 & & 26565 & & 15939 \\
\hline $\begin{array}{l}\text { Capacidad } \\
\text { disnonible }\end{array}$ & & & $\begin{array}{l}126 \\
720\end{array}$ & & $\begin{array}{l}126 \\
720\end{array}$ & & $\begin{array}{l}126 \\
720\end{array}$ & & 126 & & 126 \\
\hline
\end{tabular}

Fuente: Elaboración propia con datos tomados del almacén de la Municipalidad Provincial de

Huaura

Riesgo operacional. Se tomó en cuenta el cuestionario que se realizó a los dueños del problema puesto que de alguna manera u otra la probabilidad es tomada en la herramienta análisis de modos y efecto de fallas (AMEF) como ocurrencia, el impacto como gravedad y la detectabilidad teniendo en cuenta los controles existentes. De esta manera, se identificó según las escalas de medida cualitativa del 1 al 5 basándose en la categoría del análisis de riesgos. Así también, mediante el producto de las calificaciones dadas de la gravedad, ocurrencia y la detectabilidad se determinó el índice de prioridad de riesgo (IPR), estableciéndose de esta manera un valor límite que simbolizará el valor frontera a partir del cual la institución debe actuar. Además, el IPR máximo según la escala utilizada para todos los procesos es 125 ( 5 x 5 x 5) y el valor de IPR límite lo establece cada proceso del cual se calcula de la media de las valoraciones del IPR de todos los modos de fallo.

Tabla 8. AMEF del proceso de recepción

\begin{tabular}{|c|c|c|c|c|c|c|c|}
\hline $\begin{array}{l}\text { Actividades } \\
\text { del proceso }\end{array}$ & Modos de fallo & $\mathbf{G}$ & $\mathbf{O}$ & $\begin{array}{l}\text { Controles } \\
\text { actuales }\end{array}$ & D & IPR & IPR relativo \\
\hline \multirow{5}{*}{$\begin{array}{l}\text { Inspección y } \\
\text { verificación } \\
\text { del ingreso } \\
\text { de los } \\
\text { materiales }\end{array}$} & Faltantes de artículos & 4 & 4 & .Ninguno & 5 & 80 & $64 \%$ \\
\hline & Artículos dañados & 3 & 3 & .Ninguno & 5 & 45 & $36 \%$ \\
\hline & Artículos caducados & 4 & 4 & .Ninguno & 5 & 80 & $64 \%$ \\
\hline & $\begin{array}{l}\text { Artículos con diferentes } \\
\text { características físicas a los } \\
\text { solicitado }\end{array}$ & 3 & 3 & $\begin{array}{l}\text { Controles de } \\
\text { productos con la } \\
\text { Orden de compra }\end{array}$ & 2 & 18 & $14,40 \%$ \\
\hline & $\begin{array}{l}\text { Retraso en las actividades } \\
\text { que realiza el trabajador }\end{array}$ & 2 & 4 & .Ninguno & 5 & 40 & $32 \%$ \\
\hline
\end{tabular}

Fuente: Elaboración propia con datos de los trabaiadores de la Municipalidad Provincial de Huaura 
Continuación

\begin{tabular}{|c|c|c|c|c|c|c|c|}
\hline $\begin{array}{l}\text { Actividades } \\
\text { del proceso }\end{array}$ & Modos de fallo & $\mathbf{G}$ & $\mathbf{O}$ & Controles actuales & D & IPR & $\begin{array}{c}\text { IPR } \\
\text { relativo }\end{array}$ \\
\hline \multirow{6}{*}{$\begin{array}{c}\text { Ubicar y } \\
\text { guardar los } \\
\text { materiales } \\
\text { correctamente } \\
\text { optimizando el } \\
\text { espacio. }\end{array}$} & Pérdida de artículos & 3 & 3 & $\begin{array}{l}\text { Control de entradas y } \\
\text { salidas de artículos }\end{array}$ & 2 & 18 & $14.40 \%$ \\
\hline & Robo de artículos & 4 & 4 & .Ninguno & 5 & 80 & $64 \%$ \\
\hline & Espacio insuficiente & 4 & 4 & .Ninguno & 5 & 80 & $64 \%$ \\
\hline & $\begin{array}{c}\text { Pérdida de tiempo en } \\
\text { búsqueda }\end{array}$ & 3 & 4 & .Ninguno & 5 & 60 & $48 \%$ \\
\hline & $\begin{array}{l}\text { Inconformidad en el } \\
\text { registro de } \\
\text { entradas/salidas }\end{array}$ & 3 & 4 & $\begin{array}{l}\text { Control de entradas y } \\
\text { salidas de artículos }\end{array}$ & 2 & 24 & $19,20 \%$ \\
\hline & $\begin{array}{c}\text { Costos de } \\
\text { devaluación } \\
\text { (obsolescencia) de } \\
\text { elementos } \\
\text { desclasificados }\end{array}$ & 3 & 3 & .Ninguno & 5 & 45 & $36 \%$ \\
\hline \multirow{5}{*}{$\begin{array}{c}\text { Registrar la } \\
\text { cantidad de } \\
\text { materiales que } \\
\text { ingresan en } \\
\text { almacén y } \\
\text { realizar un } \\
\text { control de } \\
\text { inventario al } \\
\text { final del mes } \\
\text { físicamente } \\
\text { como en el } \\
\text { registro manual } \\
\text { según las } \\
\text { entradas y } \\
\text { salidas de } \\
\text { materiales. }\end{array}$} & $\begin{array}{l}\text { Error en el registro } \\
\text { de artículos }\end{array}$ & 3 & 3 & $\begin{array}{l}\text { Elaboración de } \\
\text { inventarios cada fin } \\
\text { de mes }\end{array}$ & 2 & 18 & $14,40 \%$ \\
\hline & Cálculos incorrectos & 3 & 3 & $\begin{array}{l}\text { Control de entradas y } \\
\text { salidas de artículos }\end{array}$ & 2 & 18 & $14,40 \%$ \\
\hline & $\begin{array}{c}\text { Re trabajo del } \\
\text { personal }\end{array}$ & 4 & 3 & .Ninguno & 5 & 60 & $48 \%$ \\
\hline & $\begin{array}{l}\text { Demoras en realizar } \\
\text { los requerimientos }\end{array}$ & 4 & 4 & .Ninguno & 5 & 80 & $64 \%$ \\
\hline & Faltantes de artículos & 4 & 4 & .Ninguno & 5 & 80 & $64 \%$ \\
\hline \multirow{7}{*}{$\begin{array}{l}\text { Realización de } \\
\text { la formulación } \\
\text { del pedido, } \\
\text { verificación y } \\
\text { control de } \\
\text { existencias en } \\
\text { Kardex, } \\
\text { autorización del } \\
\text { despacho, } \\
\text { Acondicionami } \\
\text { ento de los } \\
\text { materiales y } \\
\text { entrega de } \\
\text { materiales a los } \\
\text { clientes. }\end{array}$} & $\begin{array}{c}\text { Insatisfacción del } \\
\text { cliente }\end{array}$ & 4 & 3 & .Ninguno & 5 & 60 & $48 \%$ \\
\hline & $\begin{array}{l}\text { Demoras en la } \\
\text { atención de } \\
\text { materiales a los } \\
\text { clientes }\end{array}$ & 3 & 3 & .Ninguno & 5 & 45 & $36 \%$ \\
\hline & $\begin{array}{c}\text { Retraso en las } \\
\text { actividades que } \\
\text { realizan las diferentes } \\
\text { áreas }\end{array}$ & 4 & 3 & .Ninguno & 5 & 60 & $48 \%$ \\
\hline & $\begin{array}{l}\text { La nota de pedido } \\
\text { está incompleta }\end{array}$ & 4 & 3 & .Ninguno & 5 & 60 & $48 \%$ \\
\hline & $\begin{array}{l}\text { Error al elegir los } \\
\text { artículos }\end{array}$ & 4 & 4 & $\begin{array}{l}\text { Revisión de los } \\
\text { artículos pedidos }\end{array}$ & 2 & 32 & $25,60 \%$ \\
\hline & $\begin{array}{l}\text { Acondicionar el } \\
\text { pedido con mayor o } \\
\text { menor cantidad }\end{array}$ & 3 & 2 & .Ninguno & 5 & 30 & $24 \%$ \\
\hline & $\begin{array}{l}\text { Deterioro de los } \\
\text { artículos al momento } \\
\text { de ser trasladados }\end{array}$ & 3 & 3 & .Ninguno & 5 & 45 & $36 \%$ \\
\hline
\end{tabular}


En referencia al análisis AMEF elaborado, se realizó un diagrama de Pareto, el cual nos determinó el porcentaje descendente que establece cada modo de fallo dentro de cada proceso del almacén, resultando así un alto porcentaje en la clasificación A, los cuales se encuentran según el orden de índice de prioridad del riesgo, lo que indica una falta de control inmediato, ya que esto conllevaría a grandes riesgos y pérdidas económicas a la institución.

Tabla 9. Análisis de ABC de modos de fallo

\begin{tabular}{|c|c|c|c|}
\hline Modos de fallo & IPR & $\%$ acumulado & Clasificación \\
\hline Faltantes de artículos en recepción & 80 & $7 \%$ & $\mathrm{~A}$ \\
\hline Artículos caducados & 80 & $14 \%$ & A \\
\hline Robo de artículos & 80 & $21 \%$ & A \\
\hline Espacio insuficiente & 80 & $28 \%$ & A \\
\hline Demoras en realizar los requerimientos & 80 & $35 \%$ & A \\
\hline Faltantes de artículos en control de existencias & 80 & $41 \%$ & A \\
\hline Pérdida de tiempo en búsqueda & 60 & $47 \%$ & A \\
\hline Re trabajo del personal & 60 & $52 \%$ & A \\
\hline Insatisfacción del cliente & 60 & $57 \%$ & $\mathrm{~A}$ \\
\hline \multicolumn{4}{|c|}{ Continuación } \\
\hline Modos de fallo & IPR & $\%$ acumulado & Clasificación \\
\hline Retraso en las actividades que realizan las diferentes áreas & 60 & $62 \%$ & $\mathrm{~A}$ \\
\hline La nota de pedido está incompleta & 60 & $67 \%$ & A \\
\hline Artículos dañados & 45 & $71 \%$ & A \\
\hline $\begin{array}{l}\text { Costos de devaluación (obsolescencia) de elementos } \\
\text { desclasificados }\end{array}$ & 45 & $75 \%$ & A \\
\hline Demoras en la atención de materiales a los clientes & 45 & $79 \%$ & A \\
\hline
\end{tabular}

Fuente: Elaboración propia con datos tomados de los trabajadores de la Municipalidad Provincial de Huaura

Además, para solucionar los problemas encontrados dentro del análisis AMEF, se realizó un histograma del proceso general del almacén y la acumulación del IPR obtenido, resultando de la sumatoria de todos los IPR de cada actividad realizada en cada proceso. Por consiguiente, se detalló que el índice de prioridad de riesgo (IPR) obtenido con mayores IPR se encuentra en el proceso de distribución y almacenamiento, en cuanto al proceso de recepción y registro de control de existencias nos determina un bajo IPR con respecto a los otros procesos.

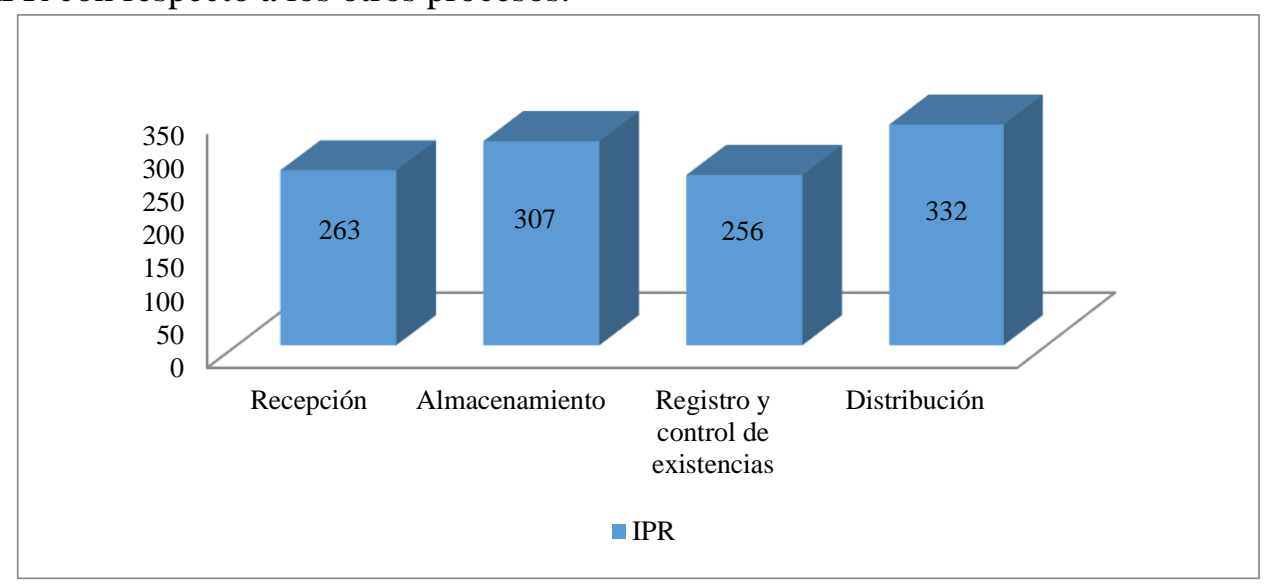

Fuente: Elaboración propia con datos de la tabla 14

Figura 1. Histograma del nivel IPR por proceso

Por otra parte, se realizó un plan de manejo del riesgo en la cual se da opciones de aceptar, evitar, transferir y reducir el riesgo. Así de esta manera minimizar o eliminar el riesgo. Además de las acciones a implantar, responsables quienes estarán a cargo de llevar a cabo dicho control, de este modo para llevar a cabo un control se propuso un tiempo de reposición de materiales y la realización de requerimientos necesarios con el modelo EOQ para productos múltiples, con esto permitir un apropiado abastecimiento de materiales y así reducir el riesgo llevando un mejor control dentro del proceso de inventario. 
Simulación. Se tomó en cuenta la muestra objeto en la cual se evaluó que artículos tienen mayor prioridad respecto al capital que se brinda. Para ello se tuvo la demanda anual desde setiembre del 2016 - agosto del 2017 y el precio unitario al que se adquirieron estos artículos dándonos así el valor total de cada producto, así mismo los gastos administrativos de los sueldos anuales de los trabajadores del área de almacén resultándonos así el capital anual al año.

Sin embargo, con la ayuda del software Crystal Ball se realizó la simulación en la cual se tomó como variable de decisión al valor total(S/.), la variable de entrada es decir la definición de las suposiciones al valor de cada producto según sea el comportamiento y gastos administrativos (sueldo del personal), así mismo como variable de salida es decir la definición del pronóstico al valor del capital.

Tabla 10: Capital total en almacén

\begin{tabular}{|c|c|c|c|}
\hline Producto & $\begin{array}{l}\text { Cantidad de } \\
\text { consumo } \\
\text { anual } \\
\text { (unid) } \\
\end{array}$ & $\begin{array}{c}\text { Precio } \\
\text { unitario } \\
(\mathrm{S} / .)\end{array}$ & $\begin{array}{l}\text { Valor Total } \\
\text { (S/.) }\end{array}$ \\
\hline Cinta Epson Fx-890 & 214 & $\mathrm{~S} / .30$ & S/.6 420 \\
\hline Tinta Epson negro & 126 & $\mathrm{~S} / .75$ & $\mathrm{~S} / .9450$ \\
\hline Tinta Epson L210 cyan & 52 & $\mathrm{~S} / .75$ & $\mathrm{~S} / .3900$ \\
\hline Tinta Epson L210 & & & \\
\hline magenta & 52 & $\mathrm{~S} / .75$ & $\mathrm{~S} / .3900$ \\
\hline $\begin{array}{l}\text { Tinta Epson L350 } \\
\text { amarillo }\end{array}$ & 51 & $\mathrm{~S} / .75$ & S/.3825 \\
\hline Papel bond A-4 & 4014 & $\mathrm{~S} / .12,40$ & $\mathrm{~S} / .49773,60$ \\
\hline Archivador & 800 & $\mathrm{~S} / .4,07$ & $\mathrm{~S} / .3256$ \\
\hline Carro de limpieza & 4 & $\mathrm{~S} / .1800$ & S/.7 200 \\
\hline \multicolumn{3}{|c|}{ Importe total } & S/.87 724,60 \\
\hline \multicolumn{4}{|c|}{ (+) Gastos administrativos } \\
\hline Concepto & & $\begin{array}{l}\text { Sueldo } \\
\text { mensual }\end{array}$ & Sueldo anual \\
\hline Jefe de almacén & & $\mathrm{S} / .2150$ & $\mathrm{~S} / .25800$ \\
\hline Personal encargado de stock & & $\mathrm{S} / .1000$ & $\mathrm{~S} / .12000$ \\
\hline \multicolumn{2}{|c|}{ Total anual } & & S/.37 800 \\
\hline & pital & & S/.125 524,60 \\
\hline
\end{tabular}

Fuente: Elaboración propia con datos del almacén de la Municipalidad Provincial de Huaura

Por otro lado, las suposiciones que se realizaron se definieron en base al comportamiento de los artículos y los gastos administrativos (sueldo del personal). Por consiguiente, para definir las previsiones o pronósticos con respecto al capital se realizó la simulación dándose así el ajuste a la distribución de probabilidad en lo que se determinó una distribución beta, es decir que los comportamientos tanto de los productos como de los gastos administrativos serán muy flexibles con respecto al capital.

Siendo así, en la figura 2 después de 10000 pruebas se obtuvo como resultado del pronóstico que la previsión del capital está entre 124 877,52 a 151 535,92 soles al 95\% de confianza y también dándonos como resultado que el promedio o media respecto al valor del capital es de 138159,20 soles y 4,90\% el coeficiente de variación, es decir que existe poca variabilidad o que los valores están cercanos al valor medio del capital. 


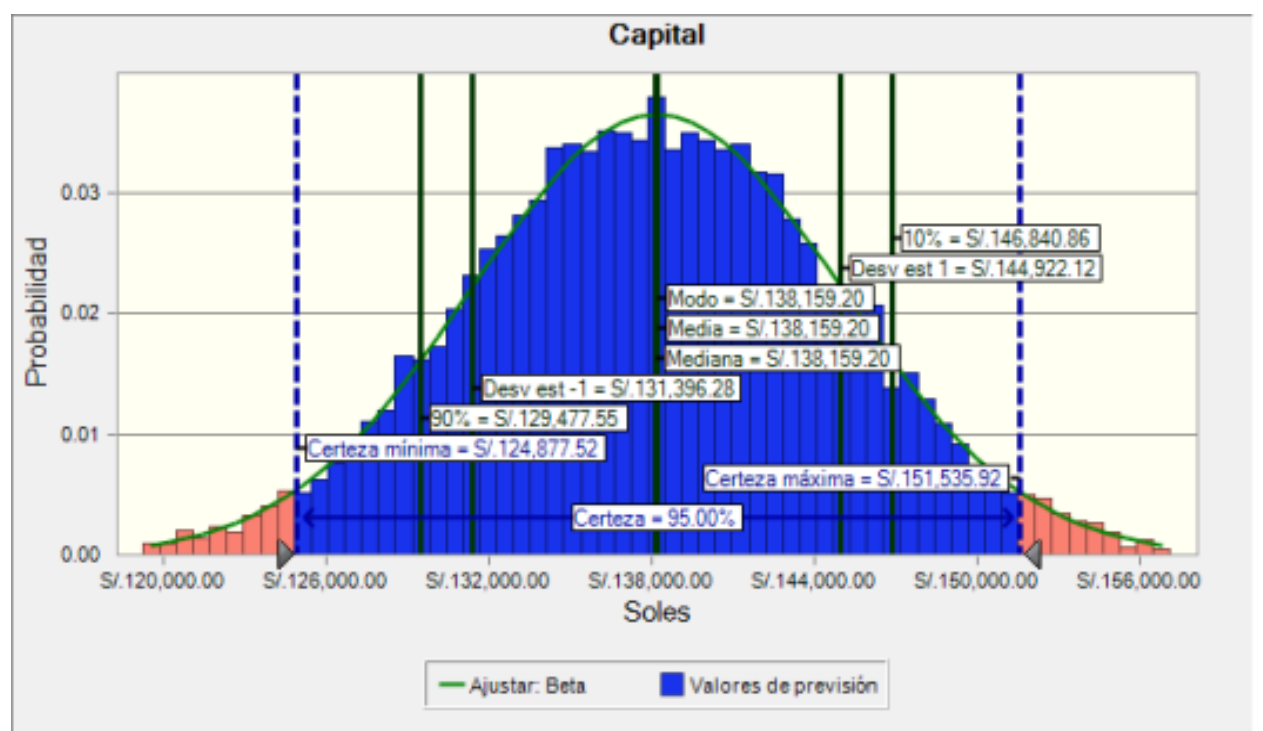

Figura 2: Previsión del capital

En otro aspecto, se obtuvo el análisis de sensibilidad de las suposiciones dadas, el cual nos permite detectar que suposiciones ya sea debido a su consumo en caso sean artículos o debido al aumento de sueldos de los personales, son a los que se tiene que dar mayor importancia y tomar decisiones para prevenir riesgos que de alguna u otra forma afectarán a los trabajadores de la institución.

De este modo, se obtuvo que el costo es sensible al papel bond A-4 quien aporta en un $86,9 \%$ en el capital representando el artículo más importante debido a su consumo como se aprecia en la figura 3.

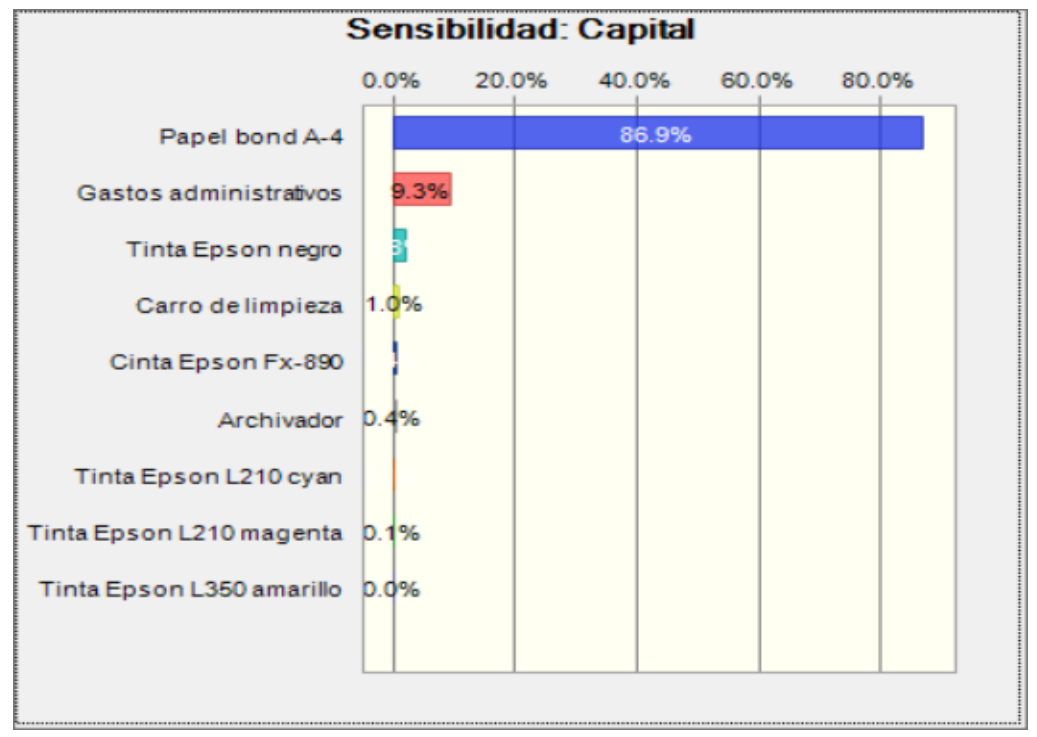

Figura 3: Análisis de sensibilidad con respecto al capital

Fuente: Software Crystal Ball

Por otro lado, se tiene los gráficos de dispersión en donde solo en el caso de papel bond A-4 se aprecia una excelente correlación $(\mathrm{R}=0,9218)$, los demás artículos y los gastos administrativos muestran una baja o débil correlación porque los datos no están agrupados como para afirmar que existe la relación. Es decir, el grado de relación existente entre el valor del papel bond A-4 y el capital es fuerte, ya que conforme el valor del papel bond A-4 aumenta, aumenta el capital, por lo que se tiene una correlación lineal positiva, como se refleja en la figura 4. 


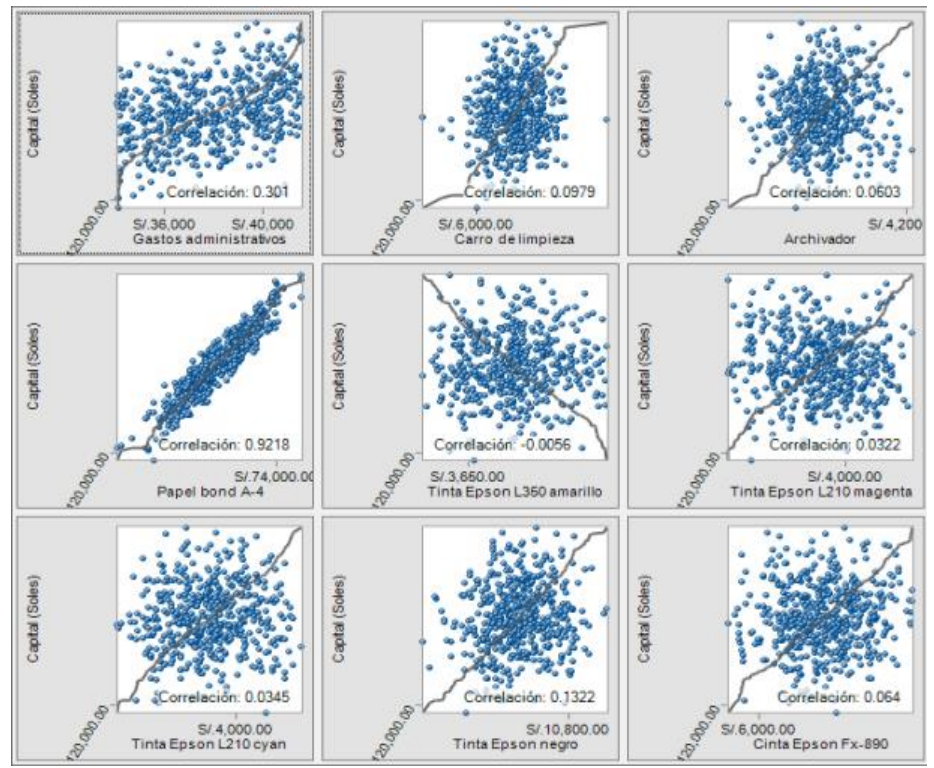

Figura 4: Gráfico de dispersión

Fuente: Software Crystal Ball

\section{Pronóstico de papel bond A-4}

Teniendo en cuenta que el artículo al que se deba de mayor importancia debido a su consumo es el papel bond A-4, se le aplicó el pronóstico para los siguientes 3 meses según la data histórica desde setiembre 2016 - agosto 2017 de la tabla 16.

Tabla 11. Data histórica del consumo de papel bond A-4

\begin{tabular}{rcc}
\hline Periodo & Mes & Demanda \\
\hline 1 & setiembre & 215 \\
2 & octubre & 238 \\
3 & noviembre & 109 \\
4 & diciembre & 234 \\
5 & enero & 0 \\
6 & febrero & 0 \\
7 & marzo & 879 \\
8 & abril & 263 \\
9 & mayo & 280 \\
10 & junio & 293 \\
11 & Julio & 1098 \\
12 & agosto & 405 \\
\hline
\end{tabular}

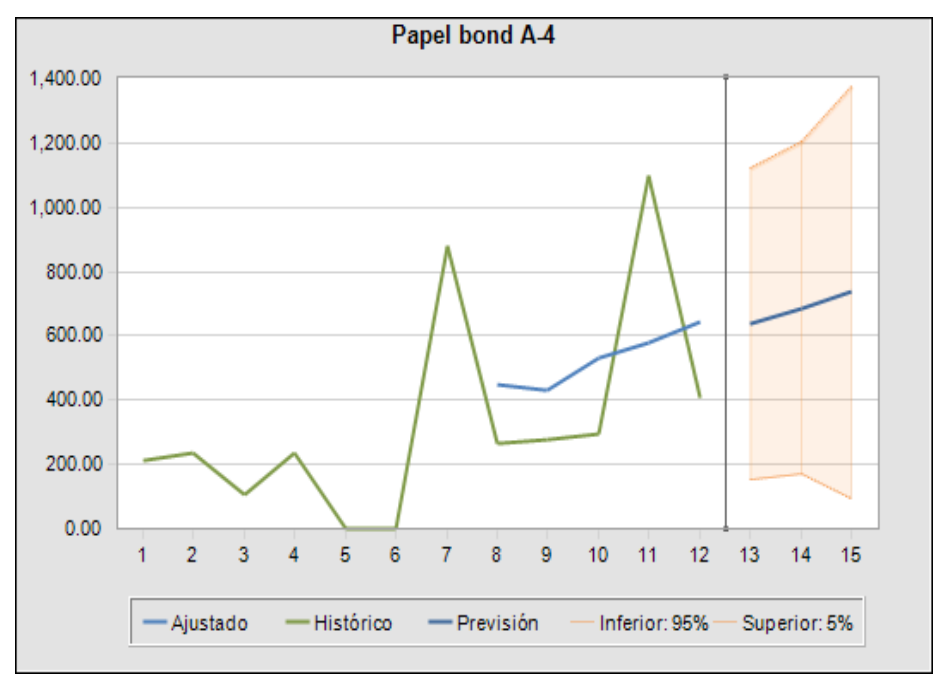

Figura 5: Gráfica del pronóstico de papel bond A-4

Fuente: Elaboración propia con datos del almacén de la MPH 
La figura 5, muestra el comportamiento gráfico de las tendencias en donde se tuvo una línea que separa los datos históricos hacia la izquierda con los datos proyectados hacia la derecha. El intervalo de confianza se aplicó para los datos proyectados al 95\% del límite inferior y al 5\% del superior como se refleja en la sombra rosada, el cual nos precisó que en la medida que aumenta el tiempo ese rango se va ir ampliando mostrando hacia el futuro el nivel de incertidumbre. Además, se detalló que el mejor método estimado de manera automática por los componentes de Crystal Ball es el promedio móvil doble el cual nos especifica una tendencia lineal puesto que este método utiliza mejor esta tendencia. Por consiguiente, la media o promedio de los valores de los datos históricos de la demanda de papel bond A-4 fue 335 millares. Además, la tendencia que se pronosticó no tiene un factor estacional debido a que existieron retrasos en los intervalos de tiempo respecto de la demanda. Por otra parte el error cuadrático medio fue de 295 millares en donde resultó de la raíz cuadrada de la diferencia o mínimos cuadrados entre los valores reales históricos y los valores de pronósticos, el cual representa la medida de desempeño respecto del pronóstico de la demanda de papel bond A-4.

Es así que, la demanda pronosticada en los 3 meses siguientes fueron: 640 millares en setiembre, 688 millares en octubre y 736 millares en noviembre del año 2017.

\section{Discusión}

En la ejecución del estudio, donde se desarrolló la variable Gestión para el control de riesgos, se ha empleado análisis de riesgos permitiendo identificar los riesgos más prioritarios dentro del proceso inventario donde se coincide con Figueroa (2015), así mismo, con la teoría de restricciones (TOC) se identificó el cuello de botella más débil en el proceso de verificación y control de existencias que al analizar los datos se observa una capacidad de 391986 minutos, la cual impide que el abastecimiento de materiales en el proceso inventario de la municipalidad sea eficiente, el cual impacta en los contribuyentes. Con el riesgo operacional al identificar los modos de fallo más críticos (AMEF) de esta forma concordamos con Consuegra (2015) y el proceso de simulación Montecarlo donde se considera que el papel bond A-4 es el articulo con mayor importancia con respecto al capital como también manifiestan Del Carpio y Eyzaguirre (2007), se obtuvo que con la aplicación del estudio permitirá abastecer de existencias a todas las áreas de la institución obteniendo a través del modelo EOQ de productos múltiples ordenados y entregados de manera conjunta un tamaño de lote alternativo siendo para la familia de artículos de oficina: 89 unidades para archivador, 559 millares para papel bond A-4 en un tiempo óptimo de 15 días; para la familia de suministros de impresora: 13 unidades para tinta Epson L210 cyan, 13 unidades para tinta Epson L210 magenta, 54 unidades para cinta Epson Fx-890, 32 unidades para tinta Epson negro y 13 unidades para tinta Epson L350 amarillo en un tiempo de 33 días. Así mismo, para la familia de artículos de limpieza: 1 carro de limpieza cada 33 días, reduciendo así 38 994,54 soles anuales del costo de mantener y ordenar inventario lo cual representa 70,47\% de ahorro que asegura la disponibilidad del nivel de existencias del proceso de inventario. Resultados similares fueron obtenidos por Bustos y Chacón (2012) en cuya investigación se logró por medio del tamaño del lote económico (EOQ) original para este caso $\mathrm{Q}=63$ resmas creando un déficit evidente para el tercer mes. Sin embargo, si adaptamos el modelo EOQ a la situación planteada mediante lotes de transferencia, encontramos un tamaño del lote económico (EOQ) de $\mathrm{Q}=155$ resmas para pedidos de papel oficio. El efecto es aumentar el tamaño Q del lote con la raíz cuadrada del número de lotes de transferencia más conveniente; en este caso, escogimos seis lotes de transferencia por ser el valor que nos proporciona el menor costo total. Así mismo, también es el caso de la investigación de Gómez, Escobar y Figueroa (2013) al aplicar un tamaño de lote con múltiples productos.

\section{Conclusiones}

Del problema general se determinó que el modelo de gestión para el control de riesgos asegurará la disponibilidad del nivel de existencias del proceso de inventario a causa de que se tiene un tamaño de lote actual que no logra abastecer a todas las áreas de la institución realizándose en un tiempo de pedido cada 6 meses siendo así, según la familia de artículos de oficina: 400 unidades para archivador y 2600 millares de papel bond A-4; según la familia de suministro de PC: 26 unidades de tinta Epson L210 cyan, 26 unidades de tinta Epson L210 magenta, 150 unidades de cinta Epson Fx-890, 78 unidades de tinta Epson negro y 26 unidades de tinta Epson L350 amarillo; según la familia de artículos de limpieza: 4 unidades de carro de limpieza se propuso una alternativa de tamaño de pedido y tiempo óptimo a través del modelo EOQ de productos múltiples ordenados y entregados de manera conjunta en la cual asegura la disponibilidad del nivel de existencias del proceso de inventario siendo lo siguiente: para la 
familia de artículos de oficina: 89 unidades para archivador, 559 millares para papel bond A-4 en un tiempo óptimo de 15 días; para la familia de suministro de PC: 13 unidades para tinta Epson L210 cyan, 13 unidades para tinta Epson L210 magenta, 54 unidades para cinta Epson Fx-890, 32 unidades para tinta Epson negro y 13 unidades para tinta Epson L350 amarillo en un tiempo de 33 días. Así mismo, para la familia de artículos de limpieza: 1 carro de limpieza cada 33 días. Además, de manera cualitativa con el cuestionario en base a la escala de Likert se concluye que el modelo de gestión para el control de riesgos asegura la disponibilidad del nivel de existencias.

\section{Referencias bibliográficas}

Álvarez, C., y Cabrera, M. (2007). Control de inventarios y su aplicación en una compañía de telecomunicaciones. Ingeniería. Investigación Y Tecnología, VII(4), 241-248.

Álvarez, J., Inche, J., y Salvador, G. (2004). Programación de Operaciones mediante la Teoría de Restricciones. Industrial Data, 7(1), 12-19.

Arango, J. A., Giraldo, J. A y Castrillón, O. D. (2013). Gestión de compras e inventarios a partir de pronósticos Holt- Winters y diferenciación de nivel de servicio por clasificación ABC. Scientia Et Technica, 18(4), 743-747. Retrieved from http://www.redalyc.org/articulo.oa?id=84929984023

Arango, M. D., Adarme, W., y Zapata, J. A. (2013). Inventarios colaborativos en la optimización de la cadena de suministros. Dyna, 80(181), 71-80.

Bustos, C. E., y Chacón, G. B. (2012). Modelos determinísticos de inventarios para demanda independiente. Un estudio en Venezuela. Contaduría Y Administración: Revista Internacional, 57(3), 239-258. Retrieved from http://www.revistas.unam.mx/index.php/rca/article/view/32169

Consuegra, O. (2015). Metodología AMFE como herramienta de gestión de riesgo en un hospital universitario. Cuadernos Latinoaméricanos de Administración, XI(20), 37-50. Retrieved from http://www.uelbosque.edu.co/sites/default/files/publicaciones/revistas/cuadernos_latinoamerican os_administracion/volumenXI_numero20_2015/05_Metodologia_Vol-X-Num-20.pdf

COSO (committee of Sponsoring Organizations of the Treadway Comission). (2004). Gestión de riesgos corporativos - Marco integrado. Técnicas de Aplicación (Permission). New York.

Del Carpio, J., y Eyzaguirre, R. (2007). Análisis de riesgo en la evaluación de alternativas de inversión utilizando Crystal Ball. Gestión Y Producción, 10(1), 55-58.

Díaz, J. A., y Pérez, D. (2012). Optimización de los niveles de inventario en una cadena de suministro. Ingeniería Industrial, XXXIII(2), 126-132. $\quad$ Retrieved from http://www.redalyc.org/articulo.oa?id=360433580004

Eppen, G. D., Gould, F. j., Schmidt, C. P., Moore, J. H., y Weatherford, L. R. (2000). Investigacion de operaciones en la ciencia de la administración (Hall, Pear). México. https://doi.org/10.1017/CBO9781107415324.004

Fernandes da Silva, G. Fossati, K. y Affonso Silva, C. (2013). TIPOS DE RIESGOS PERCIBIDOS EN EL PROCESO DE COMPRA DE BILLETES AEREOS. Estudios Y Perspectivas En Turismo, 22(1), 47-64. Retrieved from http://www.redalyc.org/articulo.oa?id=180725735003

Figueroa, L. E. (2015). Gestión de riesgos en los laboratorios clínicos, 241-250. Retrieved from http://www.redalyc.org/articulo.oa?id=96644166008

García, J., Santana, Z., Zumalacárregui, L., Quintana, M., Milá, L., Ramos, M., y Beldarraín, A. (2012). Aplicación del análisis de riesgo a la producción de proteínas recombinantes expresadas en Escherichia coli. VacciMonitor, 21(2), 35-42. Retrieved from http://www.redalyc.org/articulo.oa?id=203424333007

Gómez, J. A., Escobar, J. W., y Figueroa, Á. (2013). A Multi-Product Lot-Sizing Model for a Manufacturing Company. Ingeniería. Investigación Y Tecnología, 3(número 3), 413-419.

Gonzáles, J. A., Ortegón, K., y Rivera, L. (2003). Desarrollo de una metodología de implementación de los conceptos de TOC (Teoría de restricciones), para empresas Colombianas. Estudios Gerenciales, (87), 27-49.

González, J. C., Myer, R. A., y Pachón, W. (2017). La evaluación de los riesgos antrópicos en la seguridad corporativa: del Análisis Modal de Fallos y Efectos (AMFE) a un modelo de evaluación integral del riesgo. Revista Científica "General José María Córdova," 15(19), 269-289. Retrieved from http://www.redalyc.org/articulo.oa?id=476255361012

Heizer, J., y Render, B. (2009). Administración De Operaciones. (7ma, Ed.), Revista Latinoamericana De Administracion (Pearson Ed, Vol. 34). México. Retrieved from ISSN 1012- 8255

Hernández, N., Yelandy, M., y Cuza, B. (2013). Modelos causales para la gestión de riesgos. Revista 
Cubana de Ciencias Informáticas, 7(4), 58-74.

Izaguirre, J., y Párraga, M. del R. (2017). Refrigeradoras, Aplicación de las metodologías 8D y AMFE para reducir fallos en una fábrica de. Industrial Data, 20(2), 61-70. Retrieved from http://www.redalyc.org/articulo.oa?id=81653909009

Izar, J. M., Ynzunza, C. B., Castillo, A., y Hernández, R. (2016). Estudio comparativo del impacto de la media y varianza del tiempo de entrega y de la demanda en el costo del inventario. Ingeniería, Investigación Y Tecnología, XVII(3), 371-381.

Izar, J. M., Ynzunza, C. B., y Zermeño, E. (2015). Cálculo del punto de reorden cuando el tiempo de entrega y la demanda están correlacionados. Contaduría Y Administración, 60(4), 864-873.

Lizarzaburu, E., y Berggrun, L. (2013). Gestión del riesgo cambiario: aplicación a una empresa exportadora peruana. Estudios Gerenciales, 29(128), 379-384. Retrieved from http://www.redalyc.org/articulo.oa?id=21229786012

Llaguno, J. I. (2005). Gestión del riesgo operativo en las entidades de crédito : un camino sin retorno, 5(Basilea II), 53-77.

López, I. D., Urrea, J., y Navarro, D. (2006). Aplicación de la Teoría de Restricciones (TOC) a la gestión de facturación de las Empresas Sociales del Estado, ESE. INNOVAR. Revista de Ciencias Administrativas $Y$ Sociales, 16(27), 91-100. Retrieved from http://www.redalyc.org/articulo.oa?id=81802707

Marín, Y., Montes, J. O., Hernández, H. E., y López, J. M. (2010). Validación de la lúdica como herramienta metodológica complementaria en la enseñanza del método de producción tradicional y del método de producción de la teoría de restricciones ( TOC ) para el manejo de los entornos multitarea. Ingeniería Y Universidad, 14(1), 97-115.

Mora, J. a. N., y Gudiño, J. J. C. (2010). Riesgo operativo: esquema de gestión y modelado del riesgo. Análisis Económico, 25, 123-157. Retrieved from http://redalyc.uaemex.mx/redalyc/html/413/41313083007/41313083007_1.html

Morón, A., Reyes, M., y Urbina, Á. (2015). Gestión de riesgos en la empresa R.C Agelvis, C.A. Multiciencias, 15(4), 417-427. https://doi.org/ISSN: 1560-9146

Nápoles, A. V., Silva, Y. D., y Marrero, C. (2016). Análisis Modal de Fallo y sus Efectos de las Normas de Competencias. Ciencias Holguín, 22(2), 1-15.

Ochoa, C. M., y Rojas, B. E. (2007). Participación en plusvalías: instrumento para la gestión del riesgo en el municipio de Pereira.

Ortiz, L., y Valencia, F. J. (2017). Gestión de riesgos en eTOM. Un análisis comparativo con los estándares de riesgo corporativo. Revista LOGOS CIENCIA y TECNOLOGÍA, 9(1), 85-99. https://doi.org/http://dx.doi.org/10.22335/rlct.v9i1.334

Portuondo, Y., y Portuondo, J. (2008). Determinación de la incertidumbre de medición por el método de Monte Carlo en los procesos de manufactura. Tecnología Química, XXVIII(3), 56-62. Retrieved from http://www.redalyc.org/articulo.oa?id=445543757007

Serment, V., Mujica, H., Torres, J. A., y Welti, J. (2012). MONTE CARLO SIMULATION OF ORANGE JUICE PECTINMETHYLESTERASE (PME) INACTIVATION BY COMBINED PROCESSES OF HIGH HYDROSTATIC PRESSURE (HHP) AND TEMPERATURE. Revista Mexicana de Ingeniería Química, 11(3), 363-372. Retrieved from http://www.redalyc.org/articulo.oa?id=62026894001

Soto, A. J., Stagg, M., y Valente, M. R. (2009). Gestión del riesgo operacional en la banca universal Venezolana. Revista Venezolana de Gerencia, 14(45), 96-109.

Ulasio, A. R., y Yépez, M. (2014). Procesos de la gestión de riesgo tecnológico en consultores ambientales privados. Telos, 16(1), 31-45. Retrieved from http://www.redalyc.org/articulo.oa?id=99330402002

Valencia, M., Díaz, F. J., y Correa, J. C. (2015). Inventory planning with dynamic demand. A state of art review. Dyna, 82, 182-191. https://doi.org/10.15446/dyna.v82n190.42828

Valencia, Lambán, P., y Royo, J. (2014). Modelo analítico para determinar lotes óptimos de producción considerando diversos factores productivos y logísticos. Dyna.

Vanegas, G. A., y Pardo, C. J. (2014). Hacia un modelo para la gestión de riesgos de TI en MiPyMEs: MOGRIT. Sistemas y Telemática, 12(30), 35-48. 